\title{
Comparison of Mechanical Performance between Circular Frames and Biplanar Distraction Devices for Knee Joint Distraction
}

\author{
James MY Chowdhury ${ }^{1} \odot$, Beth Lineham², Matthew Pallett ${ }^{3}$, Hemant G Pandit ${ }^{4}$, Todd D Stewart ${ }^{5}$, Paul J Harwood ${ }^{6}$
}

\begin{abstract}
Aim and objective: This study was designed to test and compare the mechanical performance of the biplanar ArthroSave KneeReviver and a circular frame construct with the intended use of providing a mechanically favourable environment for cartilage regeneration across a knee joint. Materials and methods: Three similar constructs of the two devices were applied to biomechanical testing sawbones, with the knee distracted by $8 \mathrm{~mm}$. The constructs were vertically loaded to $800 \mathrm{~N}$ in an Instron testing machine at $20 \mathrm{~mm} / \mathrm{minute}$. Tests were conducted in neutral hip flexion and at $12^{\circ}$ of hip flexion and extension, to mimic leg position in gait. Displacement measurements were taken from the Instron machine, and three-dimensional joint motion was recorded using an Optotrak Certus motion capture system.

Results: Overall axial rigidity was similar between the two devices (circular frame, $81.6 \mathrm{~N} / \mathrm{mm} \pm 5.9$; and KneeReviver, $79.5 \mathrm{~N} / \mathrm{mm} \pm 25.1$ with hip neutral) and similar in different hip positions. At the point of joint contact, the overall rigidity of the circular frame increased significantly more than the KneeReviver $(491 \mathrm{~N} / \mathrm{mm} \pm 27$ and $93 \mathrm{~N} / \mathrm{mm} \pm 32$, respectively, $p<0.001)$. There was more variability between models in the KneeReviver. There was more off-axis motion in the KneeReviver, mainly due to increasing knee flexion on loading. This was exacerbated with the hip in flexion and extension but remained small, with the maximal off-axis displacement being $7 \mathrm{~mm} / 3^{\circ}$.

Conclusion: The circular frame provides a similar mechanical environment to the novel KneeReviver device, for which most clinical data are available. These findings suggest that both devices appear a viable option for knee joint distraction (KJD). Further clinical data will help inform mode of application.

Clinical significance: KJD is a relatively novel technique for use in osteoarthritis (OA), and it remains unclear which distraction devices provide appropriate mechanics. Our testing gives evidence to support either option for further use.

Keywords: Arthrodiastasis, Arthrodistraction, Biomechanical study, Circular frame, External fixator, Knee, Knee joint distraction, Knee osteoarthritis, Pain.

Strategies in Trauma and Limb Reconstruction (2021): 10.5005/jp-journals-10080-1530
\end{abstract}

\section{INTRODUCTION}

Osteoarthritis $(\mathrm{OA})$ is a common and disabling condition affecting an estimated 8.75 million people over the age of 45 in England. Of these, over four million people have symptomatic OA of the knee, with increasing numbers of patients presenting for treatment. ${ }^{1}$ Reasons for this are multifactorial and include an aging active population and a steadily increasing average population body mass index..$^{2-4}$ More young patients are also seeking treatment, often with the expectation of maintaining or returning to high levels of physical activity. Initial treatment is non-operative with analgesia, activity modification and physiotherapy. Whilst realignment osteotomy is appropriate in selected patients, to offload the most severely affected compartment, ${ }^{5}$ no established treatment can halt the arthritic process. When these treatments fail to provide sufficient symptomatic relief, the remaining option is a knee replacement.

Knee replacement is a proven and effective option for symptomatic OA. ${ }^{6}$ Over 100,000 knee replacements are performed in the United Kingdom per year costing over $£ 1$ billion. One in three of these patients is aged 65 years or younger. These procedures are, however, not universally successful; approximately $20 \%$ of patients report ongoing pain or dissatisfaction with their treatment. ${ }^{7}$ Furthermore, the implants have a finite lifespan, necessitating
1,2,6 Department of Trauma and Orthopaedics, Leeds General Infirmary, Leeds Teaching Hospital NHS Trust, Leeds, United Kingdom

3,5 Institute of Medical and Biological Engineering, School of Mechanical Engineering, University of Leeds, Leeds, United Kingdom

${ }^{4}$ Leeds Institute of Rheumatic and Musculoskeletal Medicine (LIRMM), Chapel Allerton Hospital, Leeds Teaching Hospital NHS Trust, Leeds, United Kingdom

Corresponding Author: James MY Chowdhury, Department of Trauma and Orthopaedics, Leeds General Infirmary, Leeds Teaching Hospital NHS Trust, Leeds, United Kingdom, Phone: +07891476489, e-mail: james.chowdhury@nhs.net

How to cite this article: Chowdhury JMY, Lineham B, Pallett M, et al. Comparison of Mechanical Performance between Circular Frames and Biplanar Distraction Devices for Knee Joint Distraction. Strategies Trauma Limb Reconstr 2021;16(2):71-77.

Source of support: Nil

Conflict of interest: None

complex, expensive revision surgery. This risk is particularly high in those receiving replacements under the age of 60 , with an average time to failure of only 4.5 years. ${ }^{8}$ Joint revision is associated with an increased cost, risk of complications and inferior outcomes. ${ }^{8-10}$

(-) Jaypee Brothers Medical Publishers. 2021 Open Access This article is distributed under the terms of the Creative Commons Attribution-Non Commercial-share alike license (https://creativecommons.org/licenses/by-nc-sa/4.0/) which permits unrestricted distribution, and non-commercial reproduction in any medium, provided you give appropriate credit to the original author(s) and the source, provide a link to the Creative Commons license, and indicate if changes were made. If you remix, transform, or build upon the material, you must distribute your contributions under the same license as original. The Creative Commons Public Domain Dedication waiver (http://creativecommons.org/publicdomain/zero/1.0/) applies to the data made available in this article, unless otherwise stated. 
It is, therefore, prudent to delay knee replacement surgery wherever possible in younger patients.

A novel approach to the treatment of $O A$ is joint distraction by external fixation, which has shown promising results in the treatment of ankle OA. ${ }^{11-16}$ Knee joint distraction (KJD) is an adaptation to this concept, used for patients with tibiofemoral OA to achieve symptomatic relief with a view to delaying the need for knee arthroplasty. Distraction is applied across the knee via percutaneous external fixation in the tibia and femur and maintained for around 6 weeks. Although the mechanism is not fully understood, it is postulated that this offloads the cartilage whilst allowing intermittent symmetrical axial motion on weightbearing, with resultant synovial fluid pressure oscillation. The resultant mechanical and biochemical environment appears to favour cartilage regeneration. ${ }^{11,17}$ KJD has been shown to achieve significant symptom improvement and restoration of cartilage with a low rate of conversion to knee replacement in a number of observational studies. ${ }^{18-20}$ However, clinical-and cost-effectiveness of KJD compared to the current gold standard of treatment, joint replacement, is unknown. A large multicentre trial investigating this is planned. ${ }^{21}$

KJD can be performed with various devices. The ArthroSave KneeReviver has been developed specifically for knee distraction and is CE marked for this purpose. ${ }^{22,23}$ This is a biplanar device comprised of two purpose-built fixators, one either side of the knee, fixed with eight standard $6 \mathrm{~mm}$ half-pins. It is the current standard for knee distraction but is not routinely used in the United Kingdom. A more familiar and widely available option might be the use of existing modular circular frame devices. Though direct implant costs are likely similar or higher than the ArthroSave, this may well be offset in the United Kingdom by indirect savings due to existing healthcare logistics. A circular fixator may produce a different mechanical environment to the established KneeReviver device, and the effect of this in KJD is unknown. The ArthroSave device incorporates an internal spring to allow motion of the construct on weight-bearing that is not available for the circular fixator. Prior to use in a clinical setting, it is important to understand the mechanical properties of the ArthroSave KneeReviver and a standard circular frame construct, which might be used for knee distraction. This simulated biomechanical study has two purposes: (1) To test the rigidity of two KJD constructs and analyse how they perform under increasing mechanical load; and (2) compare the performance of each construct to establish any significant difference between them that might alter clinical outcomes in vivo.

\section{Materials and Methods}

\section{Construction of Models for Testing}

Constructs for testing were assembled using clinical grade surgical devices implanted by the senior author, an experienced, fellowship-trained, limb reconstruction surgeon. Biomechanical grade SawBone ${ }^{\mathrm{TM}}$ anatomic left femur and tibia mechanical bone substitutes (SawBones, Vashon, Washington, United States) were used. A symmetrical $8 \mathrm{~mm}$ gap was preserved at the knee joint using spacers to ensure consistency between models. This is greater than the $5 \mathrm{~mm}$ of distraction recommended in the KneeReviver surgical technique, to allow examination of displacements beyond this, should they occur on testing. Three similar models of each device were constructed for testing.

The KneeReviver device (ArthroSave, Culemborg, Netherlands) constructs were assembled according to the surgical techniques manual with reference pins placed in the tibia and femur, parallel to the joint surfaces to align the construct in the coronal plane. Six-millimetre diameter pre-drilled, self-tapping half-pins were used throughout. The medial fixator was placed first followed by the lateral fixator (Fig. 1A).

Circular fixators were applied according to recognised techniques. ${ }^{24}$ The tibial segment consisted of two full $180 \mathrm{~mm}$ rings, each containing two wires and a half-pin. The femoral segment consisted of a $205 \mathrm{~mm}$ full ring distally with two wires and a halfpin; and a $205 \mathrm{~mm}$ half-ring proximally fixed with three half-pins, connected using femoral arch supports (Fig. 1B). Fixation elements were inserted according to recognised safe insertion corridors and were pretensioned to $1300 \mathrm{~N} .{ }^{25}$ Aluminium Taylor spatial frame rings (Smith \& Nephew Inc, Memphis, Tennessee, United States) were used.

\section{Mechanical Testing}

Models were mounted in an Instron E5980 (Instron, High Wycombe, United Kingdom) universal testing machine equipped with a $5 \mathrm{kN}$ sub-load cell. The tibia was fixed in the device distally using fixation bolts (Fig. 2). The load was applied proximally through the greater

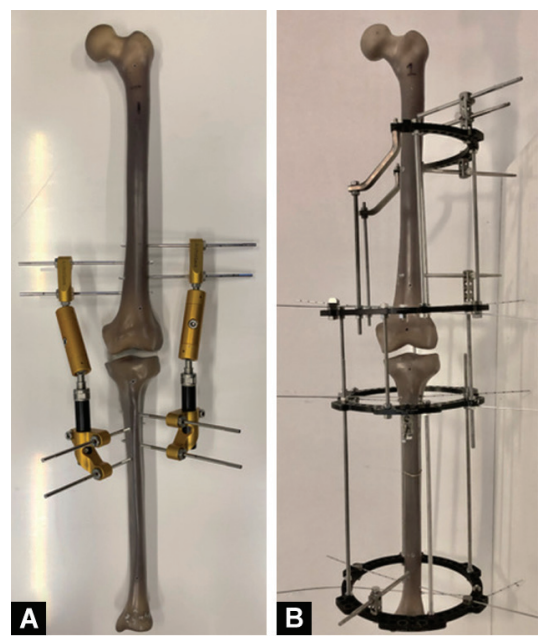

Figs $1 \mathrm{~A}$ and B:Two constructs: (A) ArthroSave KneeReviver; (B) Circular frame

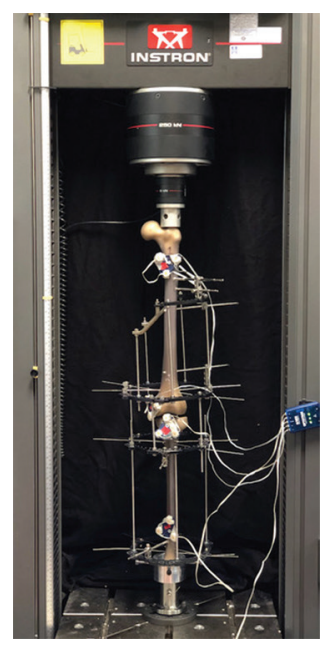

Fig. 2: Circular frame mounted in an Instron E5980 with a $5 \mathrm{kN}$ sub-load cell and Optotrak motion sensors 
trochanter, as loading via the femoral head was noted to produce significant varus. Models were preconditioned by cyclical loading prior to testing. An axial load of $800 \mathrm{~N}$ was sequentially applied to achieve vertical displacement at a rate of $20 \mathrm{~mm} /$ minute. Axial deformation of the construct was measured directly by the Instron machine. The global rigidity of each construct was calculated in $\mathrm{N} / \mathrm{mm}$, as the amount force required for $1 \mathrm{~mm}$ of axial deformation from the gradient of the linear portion of the load-displacement curves. An Optotrak Certus 3D motion capture system (NDI Europe, Bodensee, Germany) was used to measure three-dimensional displacement of the femoral and tibial bone analogues via paired infrared emitters applied to each bone segment. This allowed relative motion at the knee to be calculated with an accuracy and resolution of 0.1 and $0.01 \mathrm{~mm}$, respectively. The tests were initially undertaken with the leg vertical to simulate stance in the neutral hip position. The tests were then repeated with the distal fixation point moved off-axis anteriorly and posteriorly to achieve $12^{\circ}$ of hip extension and flexion, simulating limb position during normal gait. Each of the three models for both constructs was tested three times in each position.

\section{Data Analysis}

Data were collected and analysed using Microsoft Excel ${ }^{\circledR}$ (Microsoft Corporation, Redmond, Washington, United States). Mean overall stiffness was calculated and compared using $t$-test analysis. Global load versus deformation was calculated by comparison of the Instron and Optotrak data. A $p$-value of $<0.05$ was considered statistically significant throughout.

\section{Results}

\section{Overall Construct Rigidity}

With the hip in a neutral position, the mean overall construct rigidity of the two systems was similar (circular frame, $85 \mathrm{~N} / \mathrm{mm} \pm 3.6$; KneeReviver, $80 \mathrm{~N} / \mathrm{mm} \pm 25.1 ; p=0.15$ ). At $12^{\circ}$ hip flexion, the mean stiffness was $78.93 \mathrm{~N} / \mathrm{mm} \pm 8.11$ in the circular frame and $98.94 \mathrm{~N} / \mathrm{mm} \pm 29.32$ in the KneeReviver (mean difference, $20.01 \mathrm{~N} / \mathrm{mm} ; p=0.09$ ). At $12^{\circ}$ hip extension, the mean stiffness was $87.43 \mathrm{~N} / \mathrm{mm} \pm 5.82$ in the circular frame and $100.64 \mathrm{~N} / \mathrm{mm} \pm 21.77$ in the KneeReviver (mean difference, $13.21 \mathrm{~N} / \mathrm{mm} ; p=0.10$ ). There was greater variability in the mechanical performance of the
KneeReviver constructs between models in all tests, as reflected in the standard deviation.

On axial loading, both constructs underwent initial linear deformation as load increased (Fig. 3). At around $600 \mathrm{~N}$ in both constructs, stiffness rapidly increased corresponding to the point of articular contact at the knee when the distraction gap closed. After this occurred, the circular frame was more rigid than the KneeReviver (circular frame, $491 \mathrm{~N} / \mathrm{mm} \pm 27$; KneeReviver, $93 \mathrm{~N} / \mathrm{mm} \pm 32 ; p<0.0001)$. The circular frame became almost six times more rigid after joint contact, significantly more rigid than the KneeReviver. Following contact, the KneeReviver constructs underwent additional deformation in the form of knee flexion, whilst the circular frames remained relatively well-aligned.

\section{Articular Displacement}

From the Optotrak data, translation between the distal femur and proximal tibia tracking markers can be observed (Fig. 4). As the axial load was applied, the joint markers in both sawbones get closer and the gap at the knee is reduced, and eventually closed, demonstrated by the reducing vertical displacement in Figure 4 . It can be observed that motion is more linear in the circular frame construct, with

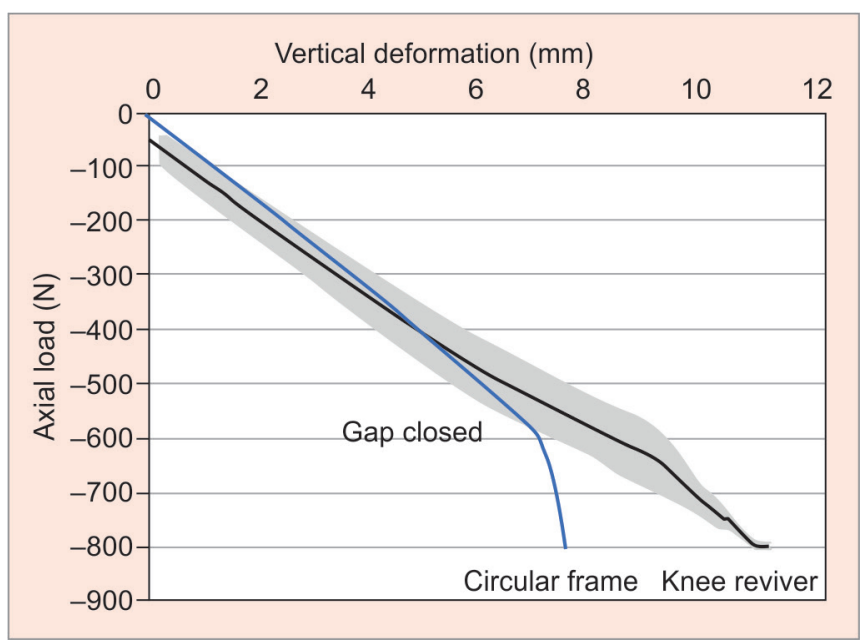

Fig. 3: Global load vs deformation, when loaded to $800 \mathrm{~N}$
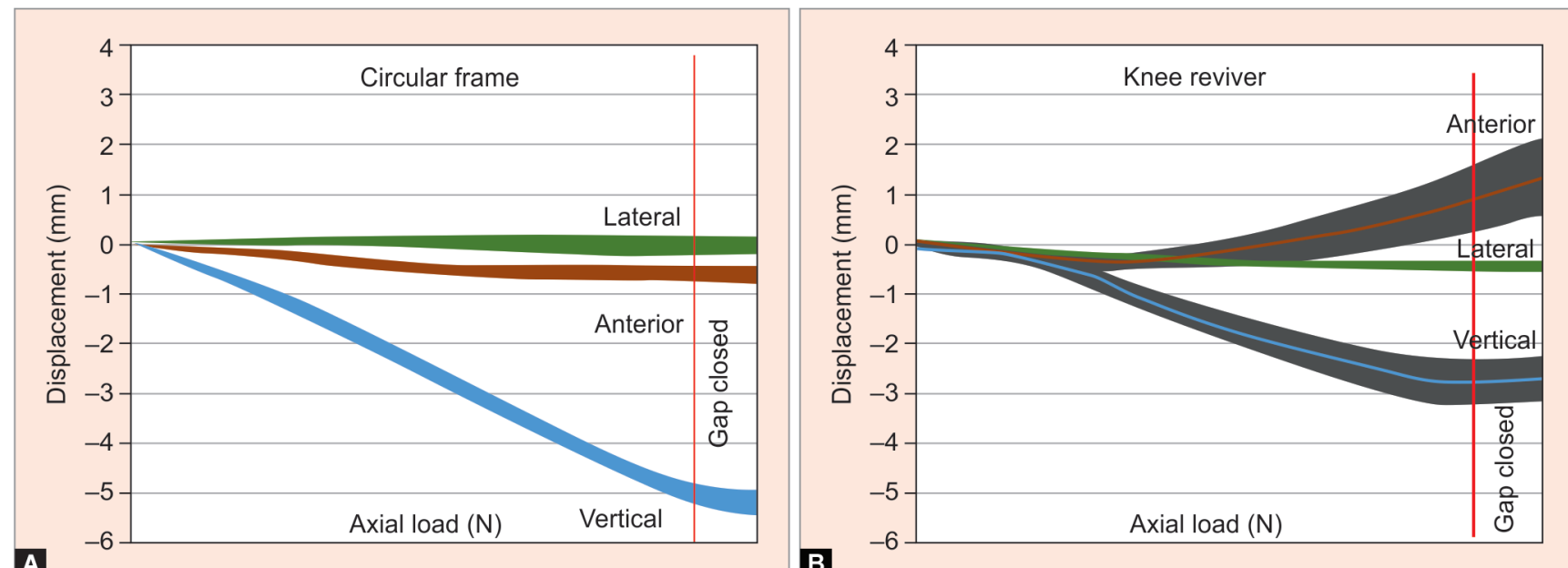

Figs 4A and B: With increasing load, articular displacement is shown: (A) Circular frame; (B) ArthroSave KneeReviver 
some anterior translation occurring in the KneeReviver. As further load is applied, global axial (vertical) displacement continues to increase; however, the articular vertical displacement ceases. In the KneeReviver models, ongoing displacement was explained by greater flexion at the knee. With this flexion, there is ongoing anterior translation, resulting in the appearance of a reduced magnitude of axial displacement. It should be noted that small angular movements of long bones result in large translations.

\section{Tibiofemoral Rotation}

Although formal testing of rotational rigidity was not performed in this study, small amounts of tibiofemoral rotation were observed in both constructs with axial load. As the constructs were loaded, the tibia tended to externally rotate on the femur in the circular frame constructs, whereas the KneeReviver demonstrated internal rotation (Table 1). Optotrak data demonstrate a high degree of variability in such small angular movements for both devices, thus preventing any strong conclusions.

\section{Knee Flexion}

Knee flexion was observed in both constructs throughout the loading process, but it was more marked in the KneeReviver device. In a neutral hip position, at the point of joint contact, the circular frame exhibited a mean knee flexion of $0.70^{\circ} \pm 0.45$, whilst $1.94^{\circ} \pm 0.76$ was exhibited by the KneeReviver (Fig. 5). At $12^{\circ}$ of hip flexion, the circular frame exhibited $-0.61^{\circ} \pm 1.16$, whilst $1.42^{\circ} \pm 0.33$ was exhibited by the KneeReviver. At $12^{\circ}$ of hip extension, the circular frame exhibited $0.42^{\circ} \pm 0.21$, whilst $1.00^{\circ} \pm 0.69$ was exhibited by the KneeReviver. The positive values denote knee flexion, whilst the negative values demonstrate extension. After the point of joint contact, as the frames are loaded beyond $600 \mathrm{~N}$, the KneeReviver flexes further, whereas the circular frame flexes less and is, therefore, more rigid (Fig. 5).

\section{Discussion}

KJD appears to be an effective and relatively low-risk management option in knee OA for younger patients. Initial data suggest similar outcomes can be achieved when compared to knee replacement or high tibial osteotomy. ${ }^{20,26,27}$ Both biplanar external fixators and circular frames are well-established orthopaedic devices that have been utilised in different types of joint distraction with documented improvement in function and increase in radiographic joint space. ${ }^{13,26-28}$ The biomechanical stability of circular fixators has been described widely in the literature. ${ }^{29,30}$ Despite the significant evidence base demonstrating the mechanical stability provided by circular frames, they have not currently been proven in KJD or directly compared with a biplanar device. Previous clinical KJD studies have used two Monotube ${ }^{\circledR}$ Triax $^{\mathrm{TM}}$ (Stryker, Kalamazoo, Michigan) proof-of-concept devices with $40 \mathrm{~kg}$ internal springs; however, there are no quantitative biomechanical data relating to this device when compared to a circular frame. ${ }^{20,26}$ Currently, there is no published literature regarding the mechanical behaviour of biplanar devices used in KJD and a paucity of data regarding circular constructs for the same purpose. Previous studies examining the use of an llizarov frame for joint distraction do not comment on the biomechanics of the frame. ${ }^{31,32}$ This study was designed to test and compare the mechanical performance of the biplanar ArthroSave KneeReviver and a circular frame construct with the intended use of providing a mechanically favourable environment for cartilage regeneration across a knee joint.

\section{Limitations}

This study has multiple limitations that should be considered when interpreting the results. The SawBone ${ }^{\mathrm{TM}}$ synthetic bone analogues used are specifically designed to replicate human anatomy and the mechanical behaviour of a normal bone. They have repeatedly been shown to be an effective bone substitutes for mechanical

Table 1: Results of tibiofemoral rotation in degrees

\begin{tabular}{lcccccc}
\hline Construct & Circularframe & KneeReviver & $\begin{array}{c}\text { Circularframe-Hip } \\
\text { flexion }\end{array}$ & $\begin{array}{c}\text { KneeReviver-Hip } \\
\text { flexion }\end{array}$ & $\begin{array}{c}\text { Circularframe-Hip } \\
\text { extension KneeReviver-Hip } \\
\text { extension }\end{array}$ \\
\hline Tibiofemoral rotation & $-1.0^{\circ} \pm 0.8$ & $0.3^{\circ} \pm 0.5$ & $-0.4^{\circ} \pm 0.4$ & $1.2^{\circ} \pm 0.6$ & $-0.2^{\circ} \pm 0.1$ & $1.1^{\circ} \pm 0.5$ \\
\hline
\end{tabular}

The negative values denote external rotation of the tibia on the femur, and the positive values demonstrate internal rotation
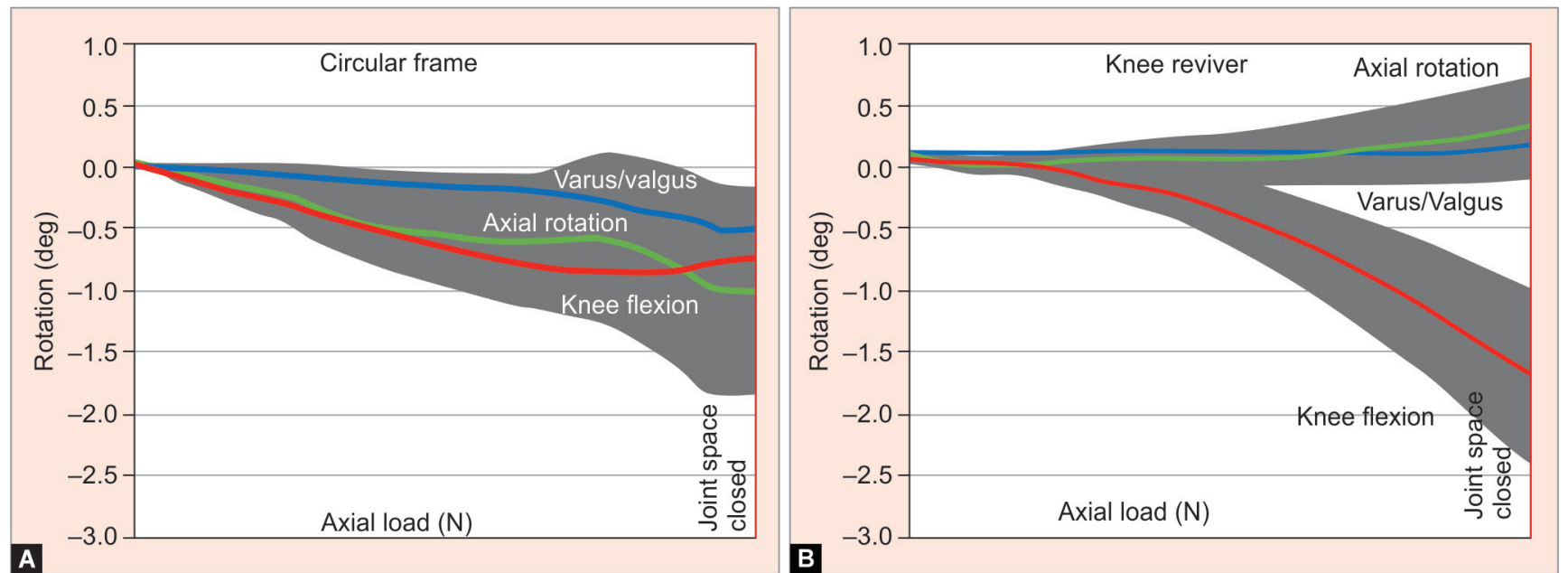

Figs 5A and B: Optotrak data demonstrating rotational movement at the knee joint with increasing load: (A) Circular frame; (B) ArthroSave KneeReviver 
testing. ${ }^{33-35}$ They carry specific advantages to cadaveric material in terms of practicality and consistency between specimens. However, disadvantages remain. Most important is the lack of soft tissue envelope in these models. These will significantly alter the forces acting across the knee and resist deformation, particularly off-axis. Whilst some translation does occur at the knee joint, any excessive motion is restricted by the presence of the cruciate ligaments, whilst the collateral ligaments prevent excessive varus/valgus movement. During a normal gait cycle, the flexion force exerted through the knee joint is counteracted by the extension force applied by the extensor mechanism. The menisci also play a role in the restriction of axial compression at the joint space. The absence of an extensor mechanism and patellofemoral joint may alter the forces loading through the knee joint. In particular, it must be considered that the additional translation observed in the KneeReviver models may be less pronounced in clinical practice due to the presence of these dynamic and static soft tissue constraints. Both devices were tested under these conditions and performed similarly, however, and comparison, therefore, appears valid.

In our study, we found that the sawbone analogues did not replicate normal anatomic alignment when the knee joint was congruent. This resulted in the mechanical axis lying significantly medial when the load was applied via the hip joint, placing a significant abnormal varus force on loading. This was overcome by transferring the proximal load plate to the tip of the greater trochanter, which loaded the knee symmetrically. Whilst a compromise from normal mechanics, it was felt that this better replicated the situation in the average patient. Clearly, mechanical alignment will vary between patients, in some of whom significant mechanical axis deviations might occur.

Although every effort was made to make each construct identical, there will be naturally be a degree of variation between constructs, and this has been reflected in the results. This will also be the case in clinical practice. For this experiment, construction of all models was undertaken by orthopaedic surgeons under the supervision of a consultant limb reconstruction specialist to ensure consistency of application.

Testing of the constructs was undertaken at the neutral hip position, $12^{\circ}$ of hip flexion and $12^{\circ}$ of hip extension to replicate normal gait, which will be undertaken in clinical situations. Normal gait requires hip flexion to $25-30^{\circ}$, so the increased hip flexion seen in clinical practice may increase deformation of the constructs, however, with a knee locked in extension hip flexion of $12^{\circ}$ is more realistic. ${ }^{36}$ Despite efforts to replicate gait, it was not possible to examine the constructs in dynamic loading and shifting between flexion and extension. It is likely that these changes in direction place different forces through the constructs, which we have not been able to identify in static loading.

Perhaps most importantly, the mechanisms behind observed clinical improvements and apparent cartilage regeneration following joint distraction have not been elucidated. It is, therefore, not possible to determine what the ideal mechanical environment might be. Whilst it might be possible to suggest this based on basic principles, this study cannot directly determine whether one device might be superior to another, but only compare their performance. The ability to build bespoke constructs with circular frame equipment is a potential advantage over the ArthroSave KneeReviver. This allows the construction of frames with different rigidities based on the clinical requirement by the use of a varying combination of fixation elements. For the purpose of this study, a knee spanning frame with femoral and tibial components of conventional length was applied. Whilst this limits direct comparability to the shorter KneeReviver constructs, it was felt this approach would be more analogous to the clinical situation by best representing a construct that most surgeons would choose to apply. In clinical practice, a smaller length circular frame might be more acceptable to the patient if it delivers an appropriate mechanical environment; however, data are not available here to demonstrate this.

\section{Overall Rigidity}

The constructs performed similarly in terms of overall axial stiffness, with no significant differences detected when tested in the different positions of hip flexion. Knee joint contact occurred at a similar load of approximately $600 \mathrm{~N}$ for both devices. Previous studies have shown the KJD using intraoperative $X$-rays but have not objectively demonstrated that complete distraction is maintained on weightbearing. The expected load through the knee in clinical scenarios of an average patient walking with full weight on the distracted limb would be well above $600 \mathrm{~N} .{ }^{37}$ Given that the distraction gap in the models was set at $8 \mathrm{~mm}$ rather than $5 \mathrm{~mm}$ as advised in clinical practice, ${ }^{22}$ these results suggest that these constructs may not fully offload the knee joint when under normal physiological loading. The additional stability provided by soft tissue in vivo, which this model does not include, may alter the maintenance of distraction by preloading the fixators when they are distracted against this soft tissue tension. Alternatively, despite contact on loading, the mechanical environment generated still results in cartilage recovery as evidenced by the clinical and radiological improvements shown in the use of the biplanar devices. ${ }^{20,26,28}$ Previous cadaveric work looking specifically at the minimum distraction gap at the ankle has been conducted; however, these results cannot be compared to those presented here given that a different joint is being examined using different techniques. ${ }^{38}$ The authors acknowledge that a completely distracted joint with zero force across it at all times may, in fact, not necessarily be required for successful clinical results. Extrapolation of our results, assuming that behaviour remains linear at higher loads, suggests that complete distraction should be maintained at $700 \mathrm{~N}$ with a distraction gap of approximately $8 \mathrm{~mm}$ and $10 \mathrm{~mm}$ for the circular frame and KneeReviver, respectively. This is a large gap and may not be tolerated or achievable in vivo when taking into account the risk to the surrounding soft tissue restraints and neurovascular structures. At higher loads, constructs containing tensioned wires may behave non-linearly, with a self-stiffening effect, meaning that complete offloading might occur with smaller distraction gaps. This study focuses predominantly on frame stability rather than the size of the distraction gap implemented. We have chosen this approach because of the limitations of the sawbone model, namely the absence of ligamentous restraints. In order to determine the minimum gap required to maintain an offloaded knee with a particular construct, further research is required and should perhaps be performed in cadaveric models, using similar techniques to previous studies. ${ }^{38}$ Interpretation of these findings is limited by the fact that the mechanisms underlying the clinical improvement observed following KJD have not been determined. Current understanding suggests cyclical axial micromotion may generate oscillatory pressure changes in the synovial fluid, favourable to cartilage regeneration. It seems likely that work in an animal model, or indeed clinical studies, would be required to elucidate the optimum mechanical environment before firm conclusions could be drawn. 
Variations in the overall rigidity between the three builds of each device were observed, despite attempts to reproduce identical constructs on application. Although every effort was made to build mechanically similar constructs, as is the case in clinical practice, the exact position of the fixation points was not formally measured. The variation of overall stiffness in the KneeReviver constructs was greater than that of the circular frame with ranges of 56.94-133.47 N/mm and $67.79-95.57 \mathrm{~N} / \mathrm{mm}$, respectively. This suggests that the performance of the KneeReviver is more sensitive to small variations in its application or that it was more difficult to apply consistently.

\section{Rotational Rigidity}

Very little axial tibiofemoral rotation was observed on testing either design, with less than $2^{\circ}$ recorded when testing in all three hip positions. Rotational rigidity is greatly affected by the length of the construct across the knee. Our data demonstrate that the circular frame is more rotationally rigid than the KneeReviver when loaded with the hip in flexion or extension, but less rigid with the hip in neutral. This rotational movement would create a sheer force on the joint when the joint is in contact. However, the clinical significance of such a small movement for such a short period of time during the gait cycle is unclear.

\section{Rigidity in the Sagittal Plane}

The motion capture data demonstrate that, on axial loading, a larger degree of knee flexion occurred in the KneeReviver models than the circular frame. The circular frame was more resistant to out-of-plane loading than the KneeReviver, likely due to its larger span and multiplanar fixation. The KneeReviver provided uniplanar fixation on the femur, whereas the circular frame wires had greater crossing angles that made it inherently more stable. Movement demonstrated at the joint space in the circular frame constructs was predominantly, and more consistently, in the axial plane, whereas the KneeReviver was much more variable.

Our results suggest that during distraction, on physiologic loading, contact may occur. When combined with knee flexion, as exhibited in the KneeReviver, part of the joint surface may be subjected to a potentially unfavourable sheer force. The circular frame is less likely to exhibit this behaviour as it resists this motion. However, in vivo, in the presence of the knee extensor mechanism, the observed anterior translation in the KneeReviver will likely reduce considerably which may mitigate this. Furthermore, as the optimal mechanical environment for cartilage regeneration is not fully understood, it is not possible to draw definite conclusions regarding this.

\section{Clinical Relevance and Conclusion}

According to the available literature, biplanar devices have demonstrated encouraging clinical outcomes following joint distraction for OA of the knee..$^{19,20,26}$ Therefore, it would appear that the mechanical environment produced by the device is adequate for the purposes of cartilage regeneration, or at the very least clinical improvement, by joint distraction..$^{19,28,39,40}$ Circular frames have not previously been tested in KJD but have been used in distraction for other purposes, for example, osteogenesis, for decades with good outcomes. ${ }^{41-43}$ Gross mechanical performance of the devices was similar, with no significant difference in overall rigidity. Whilst subtle differences between the devices were observed, the mechanisms involved in KJD have not been elucidated and the optimal mechanical environment is not understood. Based upon basic principles, however, these differences would appear favourable to the circular frame devices. In conclusion, this study suggests that the circular frame as applied is likely to provide a similar mechanical environment to the KneeReviver device and is potentially a viable option for KJD. These results will need to be confirmed in a clinical setting. Further research to determine the effect of soft tissue constraints in cadaveric models also appears warranted alongside in vivo studies to determine the optimum mechanical environment for cartilage regeneration.

The authors have received non-financial support from Smith and Nephew during the conduct of this study in the form of equipment for the experiments.

One or more of the authors (PJH) has received a grant and non-financial support from Smith and Nephew outside of the submitted work.

Professor Pandit is a National Institute for Health Research (NIHR) Senior Investigator. The views expressed in this article are those of the author(s) and not necessarily those of the NIHR, or the Department of Health and Social Care. Professor Pandit also reports that he is the chief investigator for a NIHR funded study comparing the cost- and clinical-effectiveness of KJD with knee replacement in patients with knee OA.

This study was performed at the Institute of Medical and Biological Engineering, School of Mechanical Engineering, University of Leeds, Leeds, United Kingdom.

This paper presents independent research supported by the National Institute for Health Research (NIHR) Leeds Biomedical Research Centre (BRC).

\section{OrCID}

James MY Chowdhury 이 https://orcid.org/0000-0001-8865-9202

\section{References}

1. Versus Arthritis. The state of musculoskeletal health 2019. 2020. 31-32 p. Available from: https://www.versusarthritis.org/ media/14594/state-of-musculoskeletal-health-2019.pdf.

2. Singer SP, Dammerer D, Krismer M, et al. Maximum lifetime body mass index is the appropriate predictor of knee and hip osteoarthritis. Arch Orthop Trauma Surg 2018;138(1):99-103. DOI: 10.1007/s00402017-2825-5.

3. Lawrence RC, Felson DT, Helmick CG, et al. Estimates of the prevalence of arthritis and other rheumatic conditions in the United States. Part II. Arthritis Rheum 2008;58(1):26-35. DOI: 10.1002/art.23176.

4. Service NH. Statistics on obesity, physical activity and diet. England, 2020. Available from: https://digital.nhs.uk/data-and-information/ publications/statistical/statistics-on-obesity-physical-activity-anddiet/england-2020/part-3-adult-obesity-copy\#overweight-andobesity-prevalence.

5. Brouwer RW, Huizinga MR, Duivenvoorden T, et al. Osteotomy for treating knee osteoarthritis. Cochrane Database Syst Rev 2014;2014(12): CD004019. DOI: 10.1002/14651858.CD004019.pub4.

6. Skou ST, Roos EM, Laursen MB, et al. A randomized, controlled trial of total knee replacement. N Engl J Med 2015;373(17):1597-1606. DOI: 10.1056/NEJMoa1505467.

7. Baker PN, van der Meulen JH, Lewsey J, et al. The role of pain and function in determining patient satisfaction after total knee replacement. Data from the National Joint Registry for England and Wales. J Bone Joint Surg Br 2007;89(7):893-900. DOI: 10.1302/0301620X.89B7.19091.

8. Bayliss LE, Culliford D, Monk AP, et al. The effect of patient age at intervention on risk of implant revision after total replacement of the hip or knee: a population-based cohort study. Lancet 2017;389(10077):1424-1430. DOI: 10.1016/S0140-6736(17)30059-4. 
9. Stambough JB, Clohisy JC, Barrack RL, et al. Increased risk of failure following revision total knee replacement in patients aged 55 years and younger. Bone Joint J 2014;96-B(12):1657-1662. DOI: 10.1302/0301-620X.96B12.34486.

10. Deehan DJ, Murray JD, Birdsall PD, et al. Quality of life after knee revision arthroplasty. Acta Orthop 2006;77(5):761-766. DOI: 10.1080/17453670610012953.

11. Van Valburg AA, van Roermund PM, Lammens J, et al. Can llizarov joint distraction delay the need for an arthrodesis of the ankle? A preliminary report. J Bone Joint Surg Br 1995;77(5):720-725. Available from: https://doi.org/10.1302/0301-620X.77B5.7559696.

12. Van Valburg AA, van Roermund PM, Marijnissen AC, et al. Joint distraction in treatment of osteoarthritis: a two-year follow-up of the ankle. Osteoarthritis Cartilage 1999;7(5):474-479. DOI: 10.1053/ joca.1998.0242.

13. Marijnissen AC, Van Roermund PM, Van Melkebeek J, et al. Clinical benefit of joint distraction in the treatment of severe osteoarthritis of the ankle: proof of concept in an open prospective study and in a randomized controlled study. Arthritis Rheum 2002;46(11):28932902. DOI: 10.1002/art.10612.

14. Ploegmakers JJ, van Roermund PM, van Melkebeek J, et al. Prolonged clinical benefit from joint distraction in the treatment of ankle osteoarthritis. Osteoarthritis Cartilage 2005;13(7):582-588. DOI: 10.1016/j.joca.2005.03.002.

15. Tellisi N, Fragomen AT, Kleinman D, et al. Joint preservation of the osteoarthritic ankle using distraction arthroplasty. Foot Ankle Int 2009;30(4):318-325. DOI: 10.3113/FAI.2009.0318.

16. Greenfield S, Matta KM, McCoy TH, et al. Ankle distraction arthroplasty for ankle osteoarthritis: a survival analysis. Strategies Trauma Limb Reconstr 2019;14(2):65-71. DOI: 10.5005/ jp-journals-10080-1429.

17. Van Valburg AA, van Roy HL, Lafeber FP, et al. Beneficial effects of intermittent fluid pressure of low physiological magnitude on cartilage and inflammation in osteoarthritis. An in vitro study. J Rheumatol 1998;25(3):515-520. PMID: 9517773.

18. Van der Woude JAD, Wiegant $K$, van Roermund PM, et al. Five-year follow-up of knee joint distraction: clinical benefit and cartilaginous tissue repair in an open uncontrolled prospective study. Cartilage 2017;8(3):263-271. DOI: 10.1177/1947603516665442.

19. Wiegant $K$, van Roermund PM, Intema F, et al. Sustained clinical and structural benefit after joint distraction in the treatment of severe knee osteoarthritis. Osteoarthritis Cartilage 2013;21(11):1660-1667. DOI: 10.1016/j.joca.2013.08.006.

20. Van der Woude JAD, Wiegant K, van Heerwaarden RJ, et al. Knee joint distraction compared with high tibial osteotomy: a randomized controlled trial. Knee Surg Sports Traumatol Arthrosc 2017;25(3):876886. DOI: 10.1007/s00167-016-4131-0.

21. Research NIfH. Knee arthroplasty versus joint distraction study (KARDS) for Osteoarthritis 2020. Available from: https:// fundingawards.nihr.ac.uk/award/17/122/06.

22. Van Heerwaarden RJ, Verra W. Knee joint distraction in the treatment of severe osteoarthritis. Arthroskopie 2020;33:10077. DOI: 10.1007/ s00142-020-00382-2.

23. ArthroSave. ArthroSave joint reviving medical treatment. Available from: https://www.arthrosave.com.

24. Solomin L. The basic principles of external skeletal fixation using the ilizarov and other devices. Springer Milan; 2017.

25. Nayagam S. Safe corridors in external fixation: the lower leg (tibia, fibula, hindfoot and forefoot). Strategies Trauma Limb Reconstr 2007;2(2-3):105-110. DOI: 10.1007/s11751-007-0023-7.
26. Van der Woude JA, Wiegant K, van Heerwaarden RJ, et al. Knee joint distraction compared with total knee arthroplasty: a randomised controlled trial. Bone Joint J 2017;99-B(1):51-58. DOI: 10.1302/0301620X.99B1.BJJ-2016-0099.R3.

27. Deie $M$, Ochi $M$, Adachi $N$, et al. A new articulated distraction arthroplasty device for treatment of the osteoarthritic knee joint: a preliminary report. Arthroscopy 2007;23(8):833-838. DOI: 10.1016/j. arthro.2007.02.014.

28. Intema F, Van Roermund PM, Marijnissen AC, et al. Tissue structure modification in knee osteoarthritis by use of joint distraction: an open 1-year pilot study. Ann Rheum Dis 2011;70(8):1441-1446. DOI: 10.1136/ard.2010.142364.

29. Henderson DJ, Rushbrook JL, Stewart TD, et al. What are the biomechanical effects of half-pin and fine-wire configurations on fracture site movement in circular frames? Clin Orthop Relat Res 2016;474(4):1041-1049. DOI: 10.1007/s11999-015-4652-8.

30. Bliven EK, Greinwald M, Hackl S, et al. External fixation of the lower extremities: Biomechanical perspective and recent innovations. Injury 2019;50(Suppl. 1):S10-S17. DOI: 10.1016/j.injury.2019.03.041.

31. Van Valburg AA, van Roermund PM, Marijnissen AC, et al. Joint distraction in treatment of osteoarthritis (II): effects on cartilage in a canine model. Osteoarthritis Cartilage 2000;8(1):1-8. DOI: 10.1053/ joca.1999.0263.

32. Van Roermund PM, van Valburg AA, Duivemann E, et al. Function of stiff joints may be restored by llizarov joint distraction. Clin Orthop Relat Res 1998(348):220-227. PMID: 9553556.

33. Heiner AD. Structural properties of fourth-generation composite femurs and tibias. J Biomech 2008;41(15):3282-3284. DOI: 10.1016/j. jbiomech.2008.08.013.

34. Gardner MP, Chong AC, Pollock AG, et al. Mechanical evaluation of large-size fourth-generation composite femur and tibia models. Ann Biomed Eng 2010;38(3):613-620. DOI: 10.1007/s10439-0099887-7.

35. Zdero R, Shah S, Mosli M, et al. The effect of load application rate on the biomechanics of synthetic femurs. Proc Inst Mech Eng $\mathrm{H}$ 2010;224(4):599-605. DOI: 10.1243/09544119JEIM742.

36. Lucas EM. Clinical, kinematic, and kinetic analysis of knee arthrodesis in support of the design of a novel treatment alternative [PhD Dissertation]. Clemson, USA: Clemson University; 2014.

37. Kaufman KR, An K-N, Litchy WJ, et al. Dynamic joint forces during knee isokinetic exercise. Am J Sports Med 1991;19(3):305-316. DOI: $10.1177 / 036354659101900317$.

38. Fragomen AT, McCoy TH, Meyers KN, et al. Minimum distraction gap: how much ankle joint space is enough in ankle distraction arthroplasty? HSS J 2014;10(1):6-12. DOI: 10.1007/s11420-013-9359-3.

39. Besselink NJ, Vincken KL, Bartels LW, et al. Cartilage Quality (dGEMRIC Index) following knee joint distraction or high tibial osteotomy. Cartilage 2020;11(1):19-31. DOI: 10.1177/1947603518777578.

40. Wiegant K, Intema F, van Roermund PM, et al. Evidence of cartilage repair by joint distraction in a canine model of osteoarthritis. Arthritis Rheumatol 2015;67(2):465-474. DOI: 10.1002/art.38906.

41. Green SA, Jackson JM, Wall DM, et al. Management of segmental defects by the llizarov intercalary bone transport method. Clin Orthop Relat Res 1992(280):136-142. PMID: 1611733.

42. Song HR, Cho SH, Koo KH, et al. Tibial bone defects treated by internal bone transport using the llizarov method. Int Orthop 1998;22(5):293297. DOI: $10.1007 / \mathrm{s} 002640050263$.

43. Cattaneo R, Catagni M, Johnson EE. The treatment of infected nonunions and segmental defects of the tibia by the methods of Ilizarov. Clin Orthop Relat Res 1992(280):143-152. PMID: 1611734. 